\title{
Structure and microhardness of low pressure polymerized fullerite $\mathrm{C}_{60}$
}

\author{
A. P. Isakina, S. V. Lubenets, V. D. Natsik, A. I. Prokhvatilov, \\ M. A. Strzhemechny, L. S. Fomenko, and N. A. Aksenova

\begin{abstract}
B. I. Verkin Institute for Low Temperature Physics and Engineering, National Academy of Sciences of Ukraine, 47 Lenin Ave., 310164 Kharkov, Ukraine

E-mail: isakina@ilt.kharkov.ua
\end{abstract}

A. V. Soldatov

Department of Experimental Physics, Umea University, S-90187 Umea, Sweden

E-mail: alex.soldatov@physics.umu.se

Received June 29, 1998, revised July 27, 1998

\begin{abstract}
We have carried out low-temperature x-ray diffraction studies on $\mathrm{C}_{60}$ fullerite polymerized by low quasi-hydrostatic pressure of $1.1 \mathrm{GPa}$ at $T=563 \mathrm{~K}$. It is established that at room temperature in freshly prepared samples three phases mainly coexist, viz. a compressed cubic phase with the lattice parameter $a=13.94 \AA$, an orthorhombic $\mathrm{O}^{\prime}$ phase with the lattice parameters $a=9.12 \AA, b=9.82 \AA, c=14.60 \AA$, and a rhombohedral phase of symmetry $R 3 m$ with the parameters $a=9.20 \AA$ and $c=24.27 \AA$. Mechanical grinding or annealing at $573 \mathrm{~K}$ entails depolymerization of sintered samples and restoration of the fcc structure of pristine $\mathrm{C}_{60}$. During annealing in air, intercalation of fullerite lattice by oxygen molecules occurs as well as a substantial amount of some new phase is formed, most probably with tetragonal symmetry, the chemical composition and structure of which have not been determined. The microhardness of polymerized $\mathrm{C}_{60}$ is higher than that of single crystal samples roughly four-fold at room temperature and by a factor of 2.6 at liquid nitrogen temperature. Analysis shows that polymerization and grain boundaries give contributions to the microhardness of sintered samples but we did not succeed in separating these two contributions. The temperature dependence of the microhardness of polymerized samples exhibits a jump in the vicinity of $260 \mathrm{~K}$, where pristine $\mathrm{C}_{60}$ fullerite undergoes the fcc-sc phase transition. We think that this jump is due to a partial destruction of the polymerized state under indentor as a result of shear straining in inhomogeneous stress fields. Annealing of polymerized $\mathrm{C}_{60}$ at a temperature of $573 \mathrm{~K}$, which restores the fcc phase, leads to a considerable decrease in microhardness, the hardness «jump» near the fcc-sc transition extends in temperature and shifts to lower temperatures.
\end{abstract}

PACS: 61.10.-i, 64.70.Kb, 64.70.Pf, 62.20.Fe

\section{Introduction}

Pure fullerite $\mathrm{C}_{60}$, the molecules of which are bound by weak van der Waals forces, is orientationally disordered at room temperature and normal pressure with a face-centered (fcc) lattice of space symmetry $F m 3 m$. At $T=260 \mathrm{~K}$, a phase transition occurs to a partially ordered phase with a cubic lattice of lower symmetry, $\mathrm{Pa} 3[1]$.

Exposure of thin $\mathrm{C}_{60}$ films to visible or ultraviolet radiation [2,3] as well as to high pressures and temperatures of bulk samples of $\mathrm{C}_{60}$ [4-10] results in polymerization of $\mathrm{C}_{60}$. In the polymerization process the nearest neighbour molecules of $\mathrm{C}_{60}$ connect into linear chains, four- or six-member rings by double bonds of the hexagonal faces through 2,2 cycloaddition reaction $[4,8-10]$. This gives rise to one-dimensional orthorhombic, two-dimensional tetragonal and two-dimensional rhombohedral phases, respectively. The samples can develop one or more phases depending on external factors. Qualitative analysis, which per se is not a simple problem, is quite often even more difficult because of a pronounced texturing of the separate phases [10]. The polymeric phases formed survive, when irradia- 
tion is turned off or external pressure is relieved. Also, a rather short annealing (2-3 hours) at comparatively low temperatures $(T=523 \mathrm{~K})$ leads, as a rule, to depolymerization and restoration of the fcc structure typical of pristine fullerite $\mathrm{C}_{60}$.

Despite the great number of research works on the development of polymerization technology in bulk polycrystal specimens, the properties of each of the polymeric phases, and the $P-T$ diagram of fullerite, and many other important questions still remain to be answered. In particular, these are the influence of thermobaric conditions in the preparation of sintered samples on their final phase composition and phase structures, homogeneity of the polymeric state at different stages of its formation [especially, under low pressures $(\sim 1 \mathrm{GPa})$ and at moderate temperatures up to $573 \mathrm{~K}$ ], mechanical and other physical properties.

In this paper we used powder $\mathrm{x}$-ray diffraction and structure-sensitive microindentation to study $\mathrm{C}_{60}$ fullerite samples polymerized by low pressure and then depolymerized by mechanical destruction or thermal treatment (low-temperature annealing). The structure, the lattice parameters of separate phases, the surface morphology and microhardness are studied within the range from liquid-nitrogen to room temperature.

\section{Experimental}

The samples under consideration were prepared from $99.98 \%$ pure pristine fullerite $\mathrm{C}_{60}$ by sublimation. The sample powder was subjected to hydrostatic compression up to $1.1 \mathrm{GPa}$ using the technique close to that of Ref. [11]. After that it was kept at $563 \mathrm{~K}$ for five hours, then cooled down to room temperature and the pressure was removed. As it follows from the results in Ref. [11], such a treatment must lead to polymerization of fullerite. Indirect evidence that the polymerization of the samples was fairly complete was their insolubility in solvents (for example, in benzene), where pristine $\mathrm{C}_{60}$ dissolves.

The $\mathrm{x}$-ray diffraction studies and the measurements of microhardness were carried out on fragments of the sintered materials. The flat surfaces of these samples of typical size $5 \times 5 \mathrm{~mm}$ served as the reflecting surface in the $\mathrm{x}$-ray studies. These were also used to measure microhardness at room temperature.

The initial macroscopic flat surface of a sample was actually somewhat rough. Therefore, for low temperature measurements, when there was no possibility of finding an area suitable for indenting and the pricks were to be made blindly, the sample surface was polished. The polishing was repeatedly conducted in a mechanochemical way with benzenewetted suede. The layer removed from the sample in a single run of polishing was $5-15 \mu$ thick, i.e., several times larger than the depths of indentation dips in the preceding experiment. The total number of microhardness measurements performed on a sample at different temperatures was approximately 200 .

The next cycle of measurements was carried out on samples subjected to mechanical or thermal treatment which, as suggested in [12], should produce destruction of the polymerized state. To measure the microhardness and to perform the preliminary $\mathrm{x}$-ray diffraction studies, a sinter fragment was annealing in air at $T=573 \mathrm{~K}$ for three hours. For another series of $\mathrm{x}$-ray experiments some samples were powdered and measured without any annealing or after annealing at $573 \mathrm{~K}$ for two hours.

The $\mathrm{x}$-ray diffraction experiments were made in $\mathrm{Cu} K_{\alpha}$ radiation. The polymerized samples of $\mathrm{C}_{60}$ were studied at temperatures ranging from 80 to $300 \mathrm{~K}$, the stabilization at every temperature point being better than $\pm 0.05 \mathrm{~K}$. To obtain reliable data on phase composition, phase structure, and lattice parameters at separate reference points $(80,120$, $175,293 \mathrm{~K})$, the $\mathrm{x}$-ray diffraction patterns were recorded over a wide range of reflection angles $\left(2 \Theta=8-150^{\circ}\right)$. At other temperatures the records were taken within a limited angle range covering the most intense $\mathrm{x}$-ray reflections. In addition to the reflections from the samples, the $\mathrm{x}$-ray patterns contain lines from the reference material (electrolytically pure copper). Use of the reference material made it possible to reduce the error in the lattice parameters of separate phases, which is particularly important when studying their temperature dependencies. However, because of the multiphase state of the samples studied the error in the lattice parameters of the phases observed was somewhat higher $( \pm 0.05 \%)$ than that for pristine fullerite.

The microhardness was measured in the $80-300 \mathrm{~K}$ temperature range by the technique described in Ref. [13]. The Vickers microhardness $H_{V}$ was calculated from the expression:

$$
H_{V}=1.854 P /(2 a)^{2},
$$

where $2 a$ is the diagonal length of an indentation, $P$ is the indentor load. At room temperature the indentor load was 0.01 and $0.2 \mathrm{~N}$. The temperature dependence of $H_{V}$ was studied at $P=0.1 \mathrm{~N}$. The results obtained were produced by standard statistical methods. 


\section{Results and discussion}

\subsection{X-ray diffraction studies}

Phase composition and phase structure. The range of pressures 1 to $2 \mathrm{GPa}$ and temperatures $500-650 \mathrm{~K}$ is an intermediate one as far as the polymerization conditions of fullerite $\mathrm{C}_{60}$ are concerned. Usually, several phases start to form here simultaneously (orthorhombic, tetragonal, rhombohedral, and other), which can be strongly disordered in polymer chain length and orientation. The extent of polymerization and the structure of the respective phases, in addition to temperature and pressure, are controlled by other factors, viz., hydrostaticity, the rates of heating and/or compression, exposure time at the extreme conditions, grain size, and so forth. The randomness of the forming phases are to a large measure determined [14] by the fact that the polymerization process takes place in the fcc phase where, due to the almost free molecular rotations, there are no preferable directions for establishing covalent bonds. Owing to the joint effect of all the above-enumerated factors, the orthorhombic phase that forms at relatively low pressures differs in structure and lattice parameters from that found at higher pressures (the low-pressure orthorhombic phase is now conventionally called $\mathrm{O}^{\prime}[14]$ ). We shall return to this intermediate phase when discussing our own results. According to Agafonov et al. [15], the lattice parameters of the $\mathrm{O}^{\prime}$ phase are $a=9.1 \AA, b=9.8 \AA, c=14.7 \AA$ for polycrystal samples and, according to Moret et al. [16], $a=9.14 \AA, b=9.90 \AA, c=14.66 \AA$ for single crystals.
Below we analyze our $\mathrm{x}$-ray powder diffraction results taking into account the above facts. A general view of the most typical diffraction patterns obtained at room temperatures, is shown in Fig. 1. The sample contains several crystal phases and a minor amount of highly dispersed or amorphous phase ( $10 \%$ of the total sample volume), as evidenced by the broad halo at reflection angles $2 \Theta=14-26^{\circ}$, (which corresponds to $\sin \Theta / \lambda=$ $\left.=(7-13) \cdot 10^{-2}\right)$. No detailed analysis of the dispersed phase has been made. It should be noted that the amount of this phase remained almost unchanged in all the experiments.

The coherent reflections of the diffraction pattern is formed by the scattering from at least three phases, viz. , orthorhombic $\left(\mathrm{O}^{\prime}\right)$, rhombohedral $(\mathrm{R})$, and cubic (C). At the same time we do not rule out the tetragonal $(\mathrm{T})$ phase, whose reflections in angular positions coincide partially with those from the $\mathrm{O}^{\prime}$ and $\mathrm{R}$ phases. Such a composition of the samples polymerized as indicated above corresponds to a «mix» region in the $(P, T)$ space where all the phases involved can coexist, as suggested by Sundqvist [14], on the basis of all the currently available experimental data. It is rather difficult to separate and analyze reliably every individual phase, with the exception perhaps of the cubic one, in view of the fact that a very large number of broad reflections from these phases are superimposed one on another. Among the whole set of reflections, only a few weak ones can be readily assigned as pertaining to the cubic and no other phase. Estimation of lattice parameter of this phase yields a low value, $a=13.94 \AA$, which corresponds to a lattice

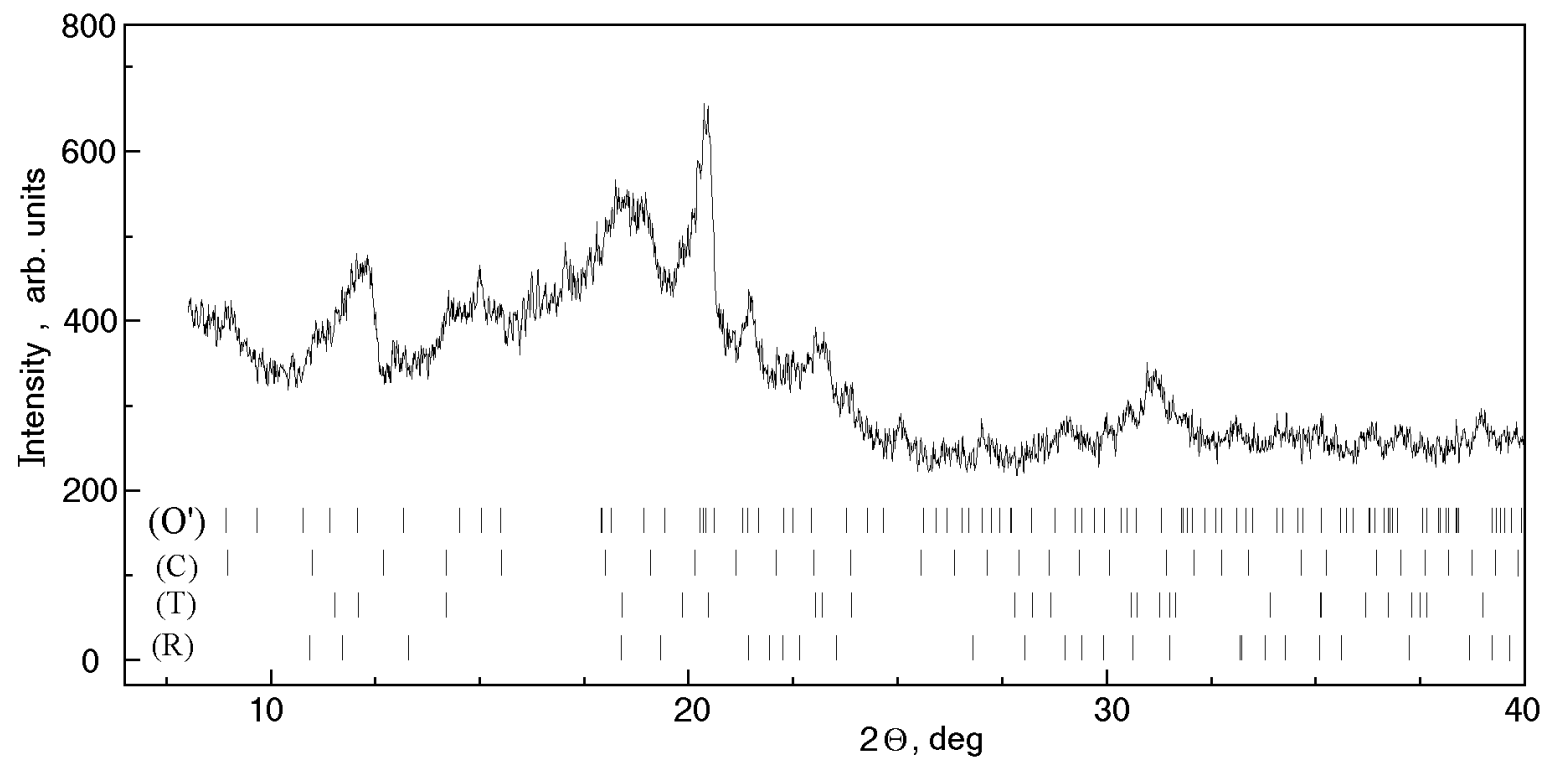

Fig. 1. Experimental powder x-ray diffraction pattern of polymerized $\mathrm{C}_{60}$ sample under study and calculated x-ray pattern (the peak positions) for the simple cubic (C), simple orthorhombic $\left(\mathrm{O}^{\prime}\right)$, tetragonal $(\mathrm{T})$ and rhombohedral $(\mathrm{R})$ of space group symmetries $I m m m$ and $R 3 m$, respectively, phases. The lattice parameters for $\mathrm{C}, \mathrm{O}^{\prime}, \mathrm{R}, \mathrm{T}$ phases are taken from Refs. $[4,8,14]$. 
strongly compressed as compared to pristine fullerite. A possibility for such a phase was previously discussed in the literature for samples subjected to high-pressure treatment $[4,5,11,14]$; this phase is presumably a partially polymerization state with randomly distributed dimers, short chains, etc. Partial polymerization leads to enhanced interaction and smaller lattice parameters; we estimate the corresponding effective interval pressure to be about $0.1 \mathrm{MPa}$.

Separation of other phases was carried out by comparing the room-temperature patterns with the line-spectra calculated for all the phases known for polymerized fullerite. The relevant line spectra are given in Fig. 1. As the source data we took calculated lattice parameters for the $\mathrm{R}$ and $\mathrm{T}$ phases from [8], for the $\mathrm{O}^{\prime}$ phase from [14], and for the cubic phase from [4]. Then the lattice parameters were refined, using the whole set of experimental reflections. The calculated line diagrams for the $\mathrm{R}$ and $\mathrm{T}$ phases were constructed using the specific models [8] for the structures of space group symmetries $R 3 \mathrm{~m}$ and $\mathrm{Immm}$, respectively, which are commonly accepted for the «high-pressure» polymeric phases. Since the space groups for both $\mathrm{C}$ and $\mathrm{O}^{\prime}$ phases have not been clearly established, the line diagrams for these phase in Fig. 1 are plotted without any absence rules. The line spectra in Fig. 1 correspond to the refined lattice parameter values, which at room temperature are: $a=9.12 \AA, b=9.82 \AA, c=$ $=14.60 \AA$ for the orthorhombic $\mathrm{O}^{\prime}$ phase, and $a=$ $=9.20 \AA, c=24.27 \AA$ for the rhombohedral phase (in hexagonal setting). These values are in reasonable agreement with the available data known in the literature for partially or completely polymerized phases. An additional indication that the polymerization of the samples was sufficiently complete was the lack of any peculiar features in the temperature dependencies of the $\mathrm{x}$-ray diffraction reflection angles or the interplane separations within the $80-293 \mathrm{~K}$ range that could be signatures of the phase transition observed in pristine $\mathrm{C}_{60}$.

A major part of the structure results presented here has previously been reported at the 1997 Joint International Meeting in Paris [17]. When indexing the diffraction pattern there we used the assumption that, in addition to the rhombohedral phase, our samples contain a tetragonal component. One of the arguments for that conclusion was the fact that the formal statistic of the r.m.s. procedure for the $\mathrm{T}$ phase was noticeably better than for the orthorhom- bic (O) one. And moreover, according to accepted notions, the $\mathrm{T}$ phase is situated much «closer» to the sample preparation conditions than the complete $\mathrm{O}$ phase. However, we must admit that our samples correspond to a certain intermediate polymerized state with far from complete phases (or phase), which are clearly understood «limit» phases with complete polymerization within planes (111) or (100), or along directions [110]. To all appearance, the «path» from the fcc phase to the tetragonal one inevitably «passes» the orthorhombic stage, the gradual crossover from the $\mathrm{O}$ to $\mathrm{T}$ phase being implied as the appearance of «closing jumpers» across the polymeric chains within plane $(100)^{*}$. The polymeric «jumpers» will be randomly distributed so that the phase will remain orthorhombic on the average, approaching the tetragonal one as the number of crossing jumpers increases.

The relative content of the polymerized phases was evaluated from the intensity ratios and the angular positions of the observed set of reflections and under the assumption of no texture in the samples under study. To within 5\% the relative amount of the $\mathrm{O}^{\prime}$ and $\mathrm{R}$ phases are 0.55:0.45.

Temperature effect. The lattice parameters of the $\mathrm{O}^{\prime}$ phase obtained at room temperature allow us to conclude that polymerization entails a considerable shrinking within the basal plane, the lattice parameter on the average diminishing by $8.9 \%$ as compared to the pristine fcc phase. Polymerization makes change the volume per molecule $\mathrm{C}_{60}$ approximately by the same quantity $(8.6 \%)$. In such hard crystals with stronger intermolecular interactions, one could expect a rather weak temperature dependence of the lattice parameters. We do not give the lattice parameters of the $\mathrm{O}^{\prime}$ phase as a function of temperature since, as mentioned before, this phase is an intermediate one exhibiting instabilities upon thermocycling. Nevertheless, it is necessary to note that the smoothed lattice parameter $a$ increases over the range $80-293 \mathrm{~K}$ as a monotonic very weak function, the net relative increment not exceeding $0.03 \%$. The lattice parameters along axes $b$ and $c$ change by $0.1 \%$ and $0.09 \%$, respectively. The elementary volume of the orthorhombic polymer $\mathrm{O}^{\prime}$ changes insignificantly with temperature: within the same temperature range the overall change is about $0.23 \%$.

The temperature dependence of the lattice parameters of the rhombohedral phase is shown in Fig. 2. The effect of temperature on the lattice

* This, in particular, can be inferred from optical measurements $[18,19]$, which give evidence that the average direction of these chains in those intermediate phases that could be termed as orthorhombic differs strongly from [110]. 

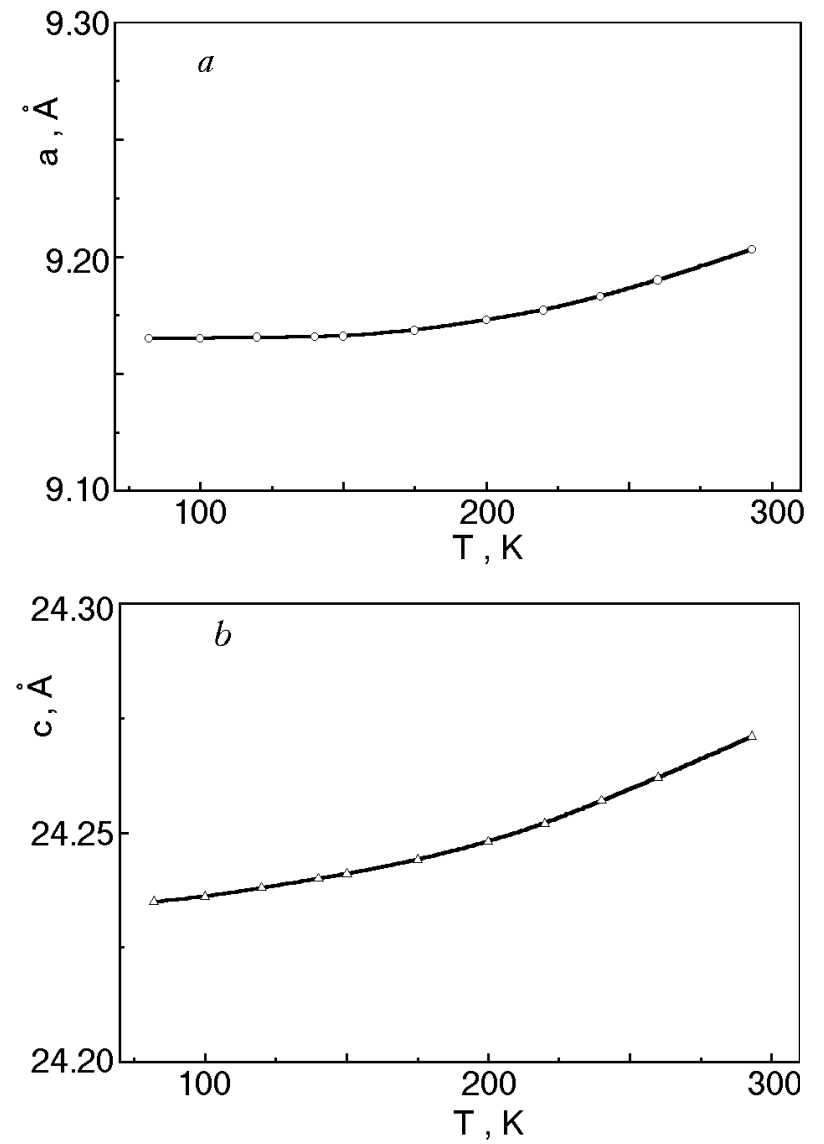

Fig. 2. Temperature dependence of the lattice parameters $a$ and $c$ (plots $a$ and $b$, respectively) of the rhombohedral phase of polymerized $\mathrm{C}_{60}$. The values of parameters are given in hexagonal setting.

parameter $a$ (and the thermal expansion in basal plane of the polymerized state) is vertually unobservable below $T \sim 150 \mathrm{~K}$ and shows a very weak tendency of expansion at higher temperatures. The overall increment of the parameter $a$ from 80 to $293 \mathrm{~K}$ does not exceed $0.2 \%$. The lattice parameter $c$ varies noticeably over the whole temperature range, especially near room temperature. Figure 3 shows relative variations of the elementary cell volumes with temperature for the rhombohedral and pristine fullerites, both normalized against the values at $80 \mathrm{~K}$. The data for the latter phase are taken from our measurements [20,21]. As seen in Fig. 3, in contrast to pristine fullerite, for which distinct discontinuities are present at the phase transition point $(260 \mathrm{~K})$ and during the orientational glassification $(T<100 \mathrm{~K})$, the volume of the $\mathrm{R}$ phase exhibits a weak uneventful increase with temperature. The overall R-phase volume change from 80 to $300 \mathrm{~K}, 0.98 \%$, although being smaller then for pristine fullerite $(5.75 \%$ [21]), nevertheless exceeds the volume increment for the $\mathrm{O}^{\prime}$ phase. This means that the thermal expansion of the $\mathrm{R}$ phase is noticeably larger than

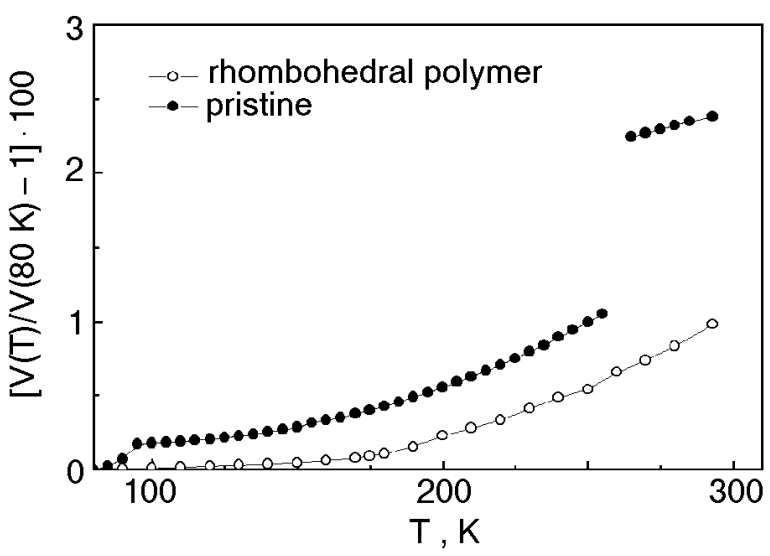

Fig. 3. Relative variation of the elementary cell volumes with temperature for the rhombohedral phase and pristine fullerite $\mathrm{C}_{60}$, both normalized against their own values $V$ at temperature $80 \mathrm{~K}$. The data for pure $\mathrm{C}_{60}$ are taken from [21].

that of $\mathrm{O}^{\prime}$. As polymerization progresses, the anharmonisms of lattice vibrations and, hence, the thermal expansion are expected [12] to decrease. The «inverse» effect obtained here for the temperaturerelated variation of the volumes of the $\mathrm{R}$ and $\mathrm{O}^{\prime}$ phases might reflect the fact that our samples were not equilibrium states, possibly owing to the peculiarities of the polymerization procedure (pressure nonhydrostaticity and relatively low temperatures). Temperature can initiate relaxation processes in such samples that provide an extra contribution to the volume increase.

Depolymerization. Mechanical grinding of a part of the initial polymerized sintered sample led to recovery of the fcc structure of pristine $\mathrm{C}_{60}$. This can be inferred from the diffraction patterns taken on powder samples. Of interest are the appreciable broadening and the low intensity of the reflections, which are typical of strongly strained samples. Subsequent annealing at $T \cong 473 \mathrm{~K}$ relieved the stresses caused by the grinding.

The structure studies on large-size sintered samples show that annealing at $573 \mathrm{~K}$ in air leads to depolymerization and formation of the equilibrium fcc phase. We present in Fig. 4 part of a diffraction pattern from an annealed sample. Note that the reflections of all the phases become appreciably narrower than in the source polymerized sample and the characteristic halo disappeared (cf. Fig. 1). We attribute these changes to the relieving of a part of inter-phase stresses and crystallization of the amorphous (or fine-grain) component. The indexing refers in Fig. 4 to the planes of the cubic (fcc) phase that re-emerges upon annealing. The respective lattice parameter, $a=14.21 \AA$, turns out to be somewhat larger then in pristine fullerite. Such an ex- 


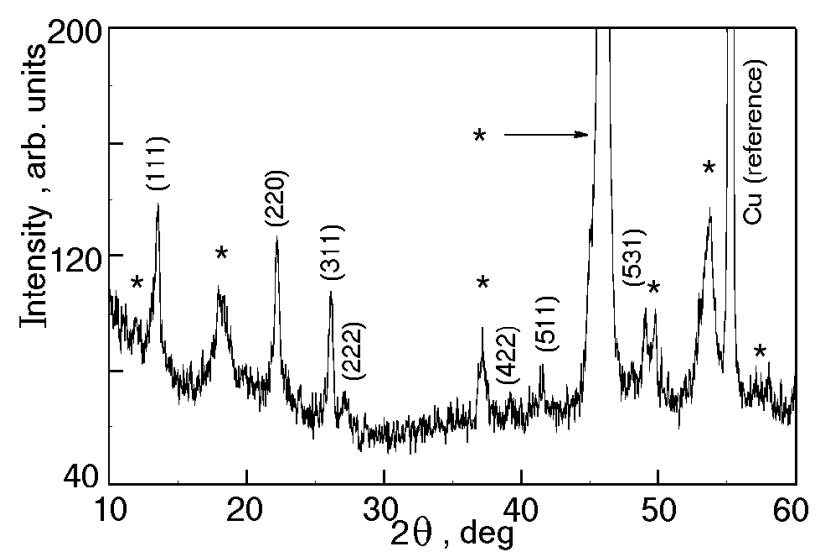

Fig. 4. X-ray diffraction pattern of depolymerized $\mathrm{C}_{60}$ after annealing at $573 \mathrm{~K}$ in air. The indices of the reflections ( $h k l)$ correspond to the $\mathrm{fcc}_{\mathrm{c}}$ phase of the initial $\mathrm{C}_{60}$ sample. The asterisks indicate reflections from the new tetragonal phase.

pansion can be due to air components penetrated into the sample during annealing, which in turn must decrease the orientational phase transition (fcc- $P(3)$ temperature. Intercalating oxygen molecules $\mathrm{O}_{2}$ are known [22] to occupy octahedral voids, whereas the transition point can vary within 3 to $20 \mathrm{~K}$, depending on the $\mathrm{O}_{2}$ concentration. As inferred from microhardness data, the orientational phase transition in annealed samples decreases by roughly $15 \mathrm{~K}$.

However, under our annealing conditions, part of the sample can interact with the air components thereby helping a new phase to form (Fig. 4). The reflections of this phase (marked with asterisks) are seen in the diffraction pattern. The nature of this new phase or compound was not determined in these experiments. Attempts to index reflections of the new phase within cubic, hexagonal, or rhombohedral lattices were unsuccessful. The best fit was attained for a tetragonal lattice with parameters $a=9.08 \AA, c=24.69 \AA$, which however does not add much to our understanding of the nature of the new phase.

Examination of annealed fragments of sintered samples as well as the measured microhardness give evidence of phase inhomogeneity which develops during annealing.

\subsection{Microhardness}

Histograms. Since the studied polymerized $\mathrm{C}_{60}$ samples are multiphase ones, we might expect a polymodal distribution of impressions in size (and microhardness). The histograms of impressions of various diagonals and microhardness based on 57 measurements at $P=0.1 \mathrm{~N}$ and $T=263-295 \mathrm{~K}$ (where temperature variations of microhardness are
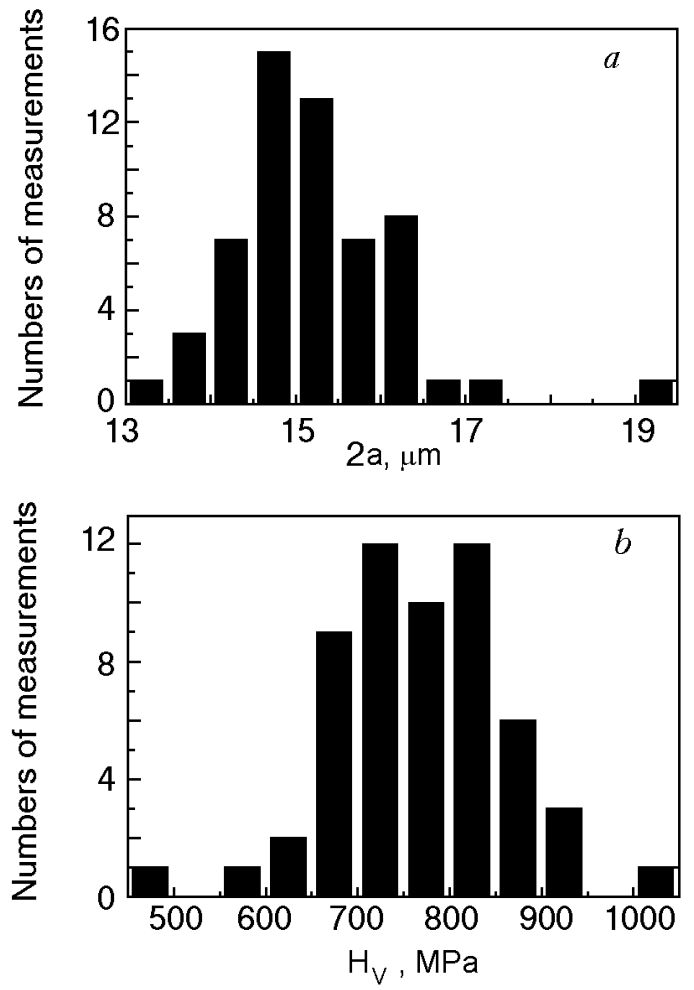

Fig. 5. Histograms for impression diagonal (a) and microhardness $(b)$ on the basis of 57 impressions on the polymerized $\mathrm{C}_{60}$ surface under a load of $P=0.1 \mathrm{~N}$ at $263-275 \mathrm{~K}$ (the temperature effect on $H_{V}$ is negligible in this range).

typically very slight) are shown in Fig. 5. It is seen that the distribution is actually single-modal. This may be because the impressions produced by the indentor were larger in size than the single phase regions. It is also seen that the relative scatter of the impression diagonal goes far beyond the measurement error $(\sim 0.15 \mu)$. The scatter may be due to the inhomogeneous structure of the sample. The relative scatter (coefficient of variation), which is the ratio of the standard deviation $s d$ to the average value, amounts to $6.3 \%$ for impression diagonals $\left(s d_{2 a}\right) \approx 0.96 \mu,(2 \bar{a})=15.2 \mu$ and $11.8 \%$ for microhardness $\left(s d_{H_{V}}=90.2 \mathrm{MPa}, \bar{H}_{V}=764.7 \mathrm{MPa}\right)$.

For single crystal $\mathrm{C}_{60}$ [20] the coefficient of microhardness variation is lower $(9 \%)$, which suggests a higher degree of inhomogeneity of our polymerized samples.

The error can be reduced appreciably (which is very important to estimation of the temperature dependence of microhardness) if we used microhardness averaged over $n$ impressions rather than its individual values. For the averaged microhardness the standard deviation decreases inversely with the number of measurements runs as $s d_{\bar{H}_{V}}=s d_{H_{V}} / n^{1 / 2}$. In Fig. $5, b, s d_{\bar{H}_{V}}=11.9 \mathrm{MPa}$ and the relative error of the average value drops to $1.6 \%$. 


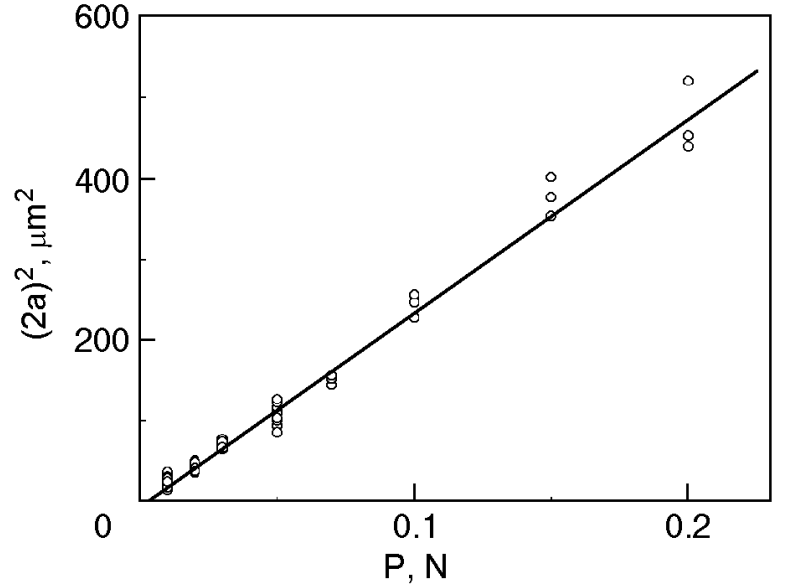

Fig. 6. Typical correlation between the squared impression diagonal and the load on indentor. The microhardness is inversely proportional to $\operatorname{tg} \alpha$ ( $\alpha$ is the angle between the straight line and the abscissa), $\bar{H}_{V}=766 \mathrm{MPa}$ (room temperature).

The temperature dependence of microhardness was studied on the surface with no fewer than 5 to 10 impressions at each temperature. The values averaged over these impressions were used to plot temperature dependencies of microhardness. At low temperatures the error of the average microhardness was two to three times higher, $\sim 3-5 \%$ (because of the smaller number of measurement runs) than it is in Fig. $5, b$.

Loading effect. The typical correlation between the load applied to the indentor and the squared diagonal of the polymerized $\mathrm{C}_{60}$ is shown in Fig. 6 . It is seen that in the range of load studied $(0-0.2 \mathrm{~N})$ microhardness was constant. Microhardness was independent of load both on the initial (non-polished) and on the polished surfaces. The average values of microhardness varied slightly after polishing the sample, i.e. the sample studied was quite homogeneous in its micromechanical properties over its depth. This helped us to derived the temperature dependence of microhardness on one sample.

Temperature effect. The temperature dependence of microhardness of polymerized $\mathrm{C}_{60}$ is shown in Fig. 7,b. For comparison, Fig. 7, $a$ shows the dependence $H_{V}(T)$ obtained earlier on single-crystalline fullerite $\mathrm{C}_{60}[20,21]$. Both the dependencies are shown in the relative coordinates in Fig. 7,c. Of interest are the following basic features.

(i). In the whole temperature range studied the microhardness of polycrystalline polymerized $\mathrm{C}_{60}$ was higher than that of nonpolymerized single crystals $\mathrm{C}_{60}: 3.8-4.5$ times higher at room temperature and 2.6 times higher at liquid nitrogen temperature. A two-fold increase in microhardness at room tempera- ture was observed by the authors [16] on $\mathrm{C}_{60}$ samples under high ( $P=2 \mathrm{GPa}$ ) pressure at $T=623 \mathrm{~K}$.

(ii). The temperature dependencies of microhardness taken on non-polymerized crystals and on polymerized samples of $\mathrm{C}_{60}$ display a sharp increase
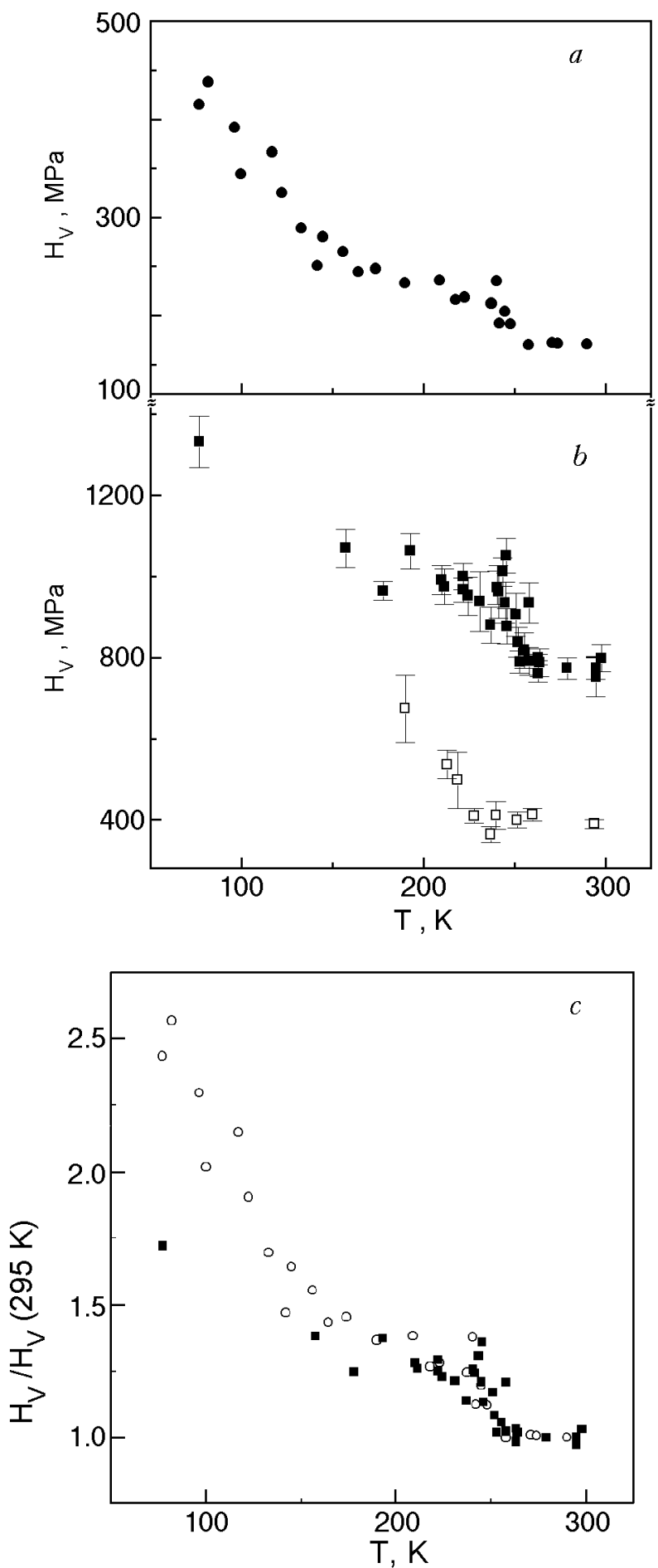

Fig. 7. Temperature dependence of the average microhardness for pure single crystal $\mathrm{C}_{60}$ (indentation in the $(100)$ plane) [20,21] (a), and polymerized ( $\square$ ) and annealed ( $\square$ ) polycrystalline $\mathrm{C}_{60}(b)$. The error bars are r.m.s. deviations for $\bar{H}_{V}$. The results for polymerized $\mathrm{C}_{60}(\boldsymbol{\square})$ and single crystal $\mathrm{C}_{60}(\mathrm{O})$ in relative units of $H_{V}(T) / H_{V}(295 \mathrm{~K})$ is shown in figure $(c)$. 
in microhardness at $240-260 \mathrm{~K}$, i.e. in the temperature region of the fcc-sc phase transition in pure $\mathrm{C}_{60}$. The fact seems to be surprising. According to $\mathrm{x}$-ray diffraction data, there is no phase transition in polymerized samples at this temperatures. The studies of acoustic and dissipative properties of the polymeric $\mathrm{C}_{60}$ phase did not detect any visible anomalies at these temperatures either [23]. This behaviour of microhardness may be attributed to destruction of the polymerized state in the region of the indentor impressions due to high stresses. This conforms with the above discussed depolymerization caused by grinding of polymerized fullerite. As shown in [24], for Si crystals under high pressures phase transitions are possible in a narrow layer of the material beneath the indentor. In the case of fullerite, depolymerization beneath the indentor might be due to non-uniform shear strains.

The slope of the microhardness versus temperature curve for polymerized samples within the 77$175 \mathrm{~K}$ range was only one half of that observed for $\mathrm{C}_{60}$ single crystals (cf. Fig. 7,c). This is in agreement with the data [23] on the temperature dependence of the elastic modulus of polymerized $\mathrm{C}_{60}$ and cubic fullerite in the low temperature region.

Annealing effect. Measurements at room temperature on a non-polished surface of annealed samples show that the average value of microhardness decreased from 765 to $440 \mathrm{MPa}$. When a thin surface layer was polished off, the value of microhardness was even lower, $H_{V}=337 \mathrm{MPa}$.

Optic microscopic observation of a polished surface of an annealed sample revealed regions of different reflectivity - light and dark. This suggests phase inhomogeneity of the sample caused by annealing. The light and dark regions differ in microhardness and have opposite $H_{V}-$ load dependencies (see Fig. 8). The latter is accounted for by the fact that under high loading onto the indentor, the impressions become comparable in size with the single-phase regions. In this case the formation of the impression is influenced by the neighboring phase.

One of the phases (the light regions) was close in microhardness to single crystalline $\mathrm{C}_{60}$. According to $\mathrm{x}$-ray diffraction data, this is the ordinary fcc phase. The other phase (the dark regions), still not identified, was harder. The temperature dependence of the average microhardness of sample also changed after annealing: the region of growing $H_{V}$ shifted towards low temperatures (Fig. 7,b). It should be noted that securing data for each of the two phases over all the temperature interval is very difficult.

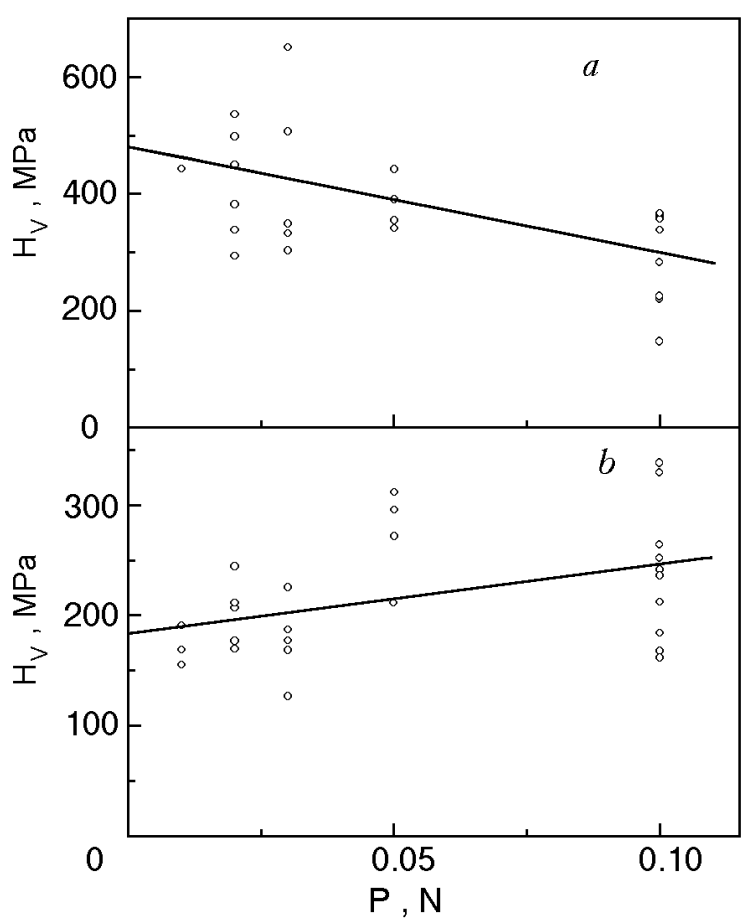

Fig. 8. The effect of load on the microhardness of two different phases formed after three hour annealing of a polymerized sample in air at $573 \mathrm{~K}$ : undentified phase $(a)$, fcc phase of $\mathrm{C}_{60}(b)$.

The higher microhardness of polymerized polycrystalline $\mathrm{C}_{60}$ can be attributed to two factors the fine-grained structure and polymerization. It is known that the correlation between the yield point $\sigma_{p}$ of a polycrystal and the grain size $d$ is well described by the Hall-Petch relation [25]:

$$
\sigma_{p}=\sigma_{0}+K_{\sigma} d^{-0.5}
$$

where $\sigma_{0}$ is the yield stress of a single crystal, and $K_{\sigma}$ is the material constant. According to Friedel [26], $K_{\sigma} \approx 0.2 \mu b^{0.5}$ for brittle materials ( $\mu$ is the shear modules, $b$ is the Burgers vector). Friedel's estimate agrees well with experimental results taken on polycrystals of some fcc metals [27]. Hall assumes [28] that the dependence of microhardness on the grain size $d$ can be described with a similar relation:

$$
H_{V}=H_{V_{0}}+K_{H} d^{-0.5},
$$

where $H_{V_{0}}$ is the microhardness of a single crystal, $K_{H}$ is the material constant. The measurement shows [29] that the dependence (3) holds for $\mathrm{Al}$, $\mathrm{Cu}$ and their alloys. The constant $K_{H}$ is three to five times higher than $K_{\sigma}$ [29].

Using the above relations and the values of microhardness measured on poly- and single crystals of $\mathrm{C}_{60}$, we can estimate the grain size ensuring the difference postulated in the assumption: the higher 
hardness of the polycrystal $\mathrm{C}_{60}$ is only due to the hardening effect of the grain structure. For $\mathrm{C}_{60}$ crystals only Young's modulus and the bulk modulus were found experimentally: $E=20 \mathrm{GPa}$ [30], $B=12-14$ GPa $[31,32]$.

Hereafter, the estimation is based on the value $B=12 \mathrm{GPa}$. The shear modulus can be calculated from $\mu=E /(3-E / 3 B) \approx 8.2 \mathrm{GPa}$. Estimation from Friedel's results gives $K_{\sigma} \sim 0.5 \mathrm{MPa} \cdot \mathrm{cm}^{0.5}$ and $K_{H} \approx 4 K_{\sigma} \approx 2 \mathrm{MPa} \cdot \mathrm{cm}^{0.5}$. To obtain the experimentally observed difference in microhardness $\left(\Delta H_{V} \approx\right.$ $\approx 600 \mathrm{MPa}$ ) of poly- and single crystals, the grain size should be $d=\left(K_{H} / \Delta H_{V}\right)^{2} \approx 10^{-5} \mathrm{~cm}$. This corresponds to the lower limit of grain sizes in $\mathrm{x}$-ray diffraction studies on polycrystal samples.

Note that $K_{\sigma}$ was calculated by the formula for brittle materials. For plastic $\mathrm{C}_{60}$ the $K_{\sigma}$ value may prove overestimated. Then, the grains ensuring the above difference in microhardness of poly- and single crystals should be even smaller.

The above analysis shows that fine-grained structure and polymerization contribute to hardening of fullerite subjected to thermobaric treatment. So far, it is hardly possible to separate the effect of these two factors. It is pertinent to note that possible depolymerization beneath the indentor makes a noncontrollable contribution to hardness.

The authors are sincerely indebted to Prof. B. Sundqvist for kindly reading the manuscript and giving several important remarks that substantially improved this paper.

The authors, S.V.L., V.D.N., and L.S.F., wish to thank the Swiss National Science Foundation for the partial financial support of this work through a program CEEC/NIS, project No 7UKPJ04864, A. V. S. acknowledges the financial support of the Swedish Natural Sciences Research Council (NFR).

1. J. D. Axe, S. C. Mooss, and D. A. Neumann, Structure and Dynamics of Crystalline $\mathrm{C}_{60}$, in: Solid State Physics, H. Ehrenreich and F. Spaepen (eds.), Academic Press, New York (1994), 48, p. 150.

2. A. M. Rao, P. Zhou, K.-A. Wang, G. T. Hager, J. M. Holden, Y. Wang, W.-T. Lee, X.-X. Bi, P. C. Eklund, D. S. Cornett, M. A. Duncan, and I. J. Amster, Science $\mathbf{2 5 9}$, 955 (1993)

3. Fullerenes and Fullerene Polymer Composites, P. C. Eklund and A. M. Rao (eds.), Springer Verlag, New York (1997).

4. Y. Iwasa, T. Arima, R. M. Fleming, T. Siegrist, O. Zhou, R. C. Haddon, L. J. Rothberg, K. B. Lyons, H. L. Carter Jr., A. F. Hebard, R. Tycko, G. Dabbagh, J. J. Krajewski, G. A. Thomas, and T. Yagi, Science 264, 1570 (1994).

5. I. O. Bashkin, V. I. Raschupkin, N. P. Kobelev, A. P. Moravsky, Ya. M. Soifer, and E. G. Poniatovsky, Pis'ma Zh. Eksp. Teor. Fiz. 59, 258 (1994) [JETP Lett. 59, 279 (1994)].
6. I. O. Bashkin, V. I. Rashchupkin,A. F. Gurov, A. P. Moravsky, O. G. Rybchenko, N. P. Kobelev, Ya. M. Soifer, and E. G. Ponyatovsky, J. Phys.: Condens. Mater. 6, 7491 (1994)

7. A. P. Moravsky, G. E. Abrosimova, I. O. Abrosimova, I. O. Bashkin, R. A. Dilanian, A. F. Gurov, N. P. Kobelev, V. I. Rashchupkin, O. G. Rybchenko, Ya. M. Soifer, V. Sh. Shekhtman, and E. G. Ponyatovsky, in: Recent $A d$ vances in the Chemistry and Physics of Fullerenes and Related Materials, R. S. Ruoff and K. M. Kadish (eds.), The Electrochemical Society, Inc., Pennington (1995), 9510, p. 952.

8. M. Nunes-Regueiro, L. Marques, J.-L. Hodeau, O. Bethoux, and M. Perroux, Phys. Rev. Lett. 74, 278 (1995).

9. L. Marques, M. Nunez-Regueiro, and M. Perroux, Phys. Rev. B54, R12633 (1996).

10. A. M. Rao, P. C. Eklund, U. D. Venkateswaran, J. Tucker, M. A. Duncan, G. Bendele, P. W. Stephens, J.-L. Hodeau, L. Marques, M. Nunez-Regueiro, I. O. Bashkin, E. G. Ponyatovsky, and A. P. Morovsky, Appl. Phys. A64, 231 ( 1997)

11. P.-A. Persson, U. Edlund, P. Jacobsson, D. Johnels, A. Soldatov, and B. Sundqvist, Chem. Phys. Lett. 258, 540 (1996).

12. B. Sundqvist, O. Anderson, U. Edlund, A. Fransson, A. Inaba, P. Jacobsson, D. Johnels, P. Launois, C. Meingast, R. Moret, T. Moritz, P. A. Persson, A. Soldatov, and T. Wagberg, in: Recent Advances in the Chemistry and Physics of Fullerenes and Related Materials, R. S. Ruoff and K. M. Kadish (eds.), The Electrochemical Society, Pennington, Inc. (1996), 96-10, p. 1014.

13. B. Ya. Farber, N. S. Sidorov, V. I. Kulakov, Yu. A. Iunin, A. N. Izotov, G. A. Emel'chenko, V. S. Bobrov, L. S Fomenko, V. D. Natsik, and S. V. Lubenets, Sverkhprovodimost' 4, 2393 (1991) (in Russian).

14. B. Sundqvist, Fullerenes under High Pressures (review), Adv. Phys. (1998), in press.

15. V. Agafonov, V. A. Davydov, L. S. Kashevarova, A. V. Rakhmanina, A. Kahn-Harari, P. Dubois, R. Ceolin, and H. Szwarc, Chem. Phys. Lett. 267, 193 (1997).

16. R. Moret, P. Launois, P.-A. Persson, and B. Sundqvist, Europhys, Lett. 40, 55 (1997).

17. N. A. Aksenova, A. P. Isakina, A. I. Prokhvatilov, M. A. Strzhemechny, A. V. Soldatov, and B. Sundqvist, in: $R e^{-}$ cent Advances in the Chemistry and Physics of Fullerenes and Related Materials, R. S. Ruoff and K. M. Kadish (eds.), The Electrochemical Society, Inc., Pennington (1997), 97-42, p. 687 .

18. V. A. Davydov, L. S. Kashevarova, A. V. Rakhmanina, A. V. Dzyabchenko, V. Agafonov, P. Dubois, R. Ceolin, and H. Szwarc, Pis'ma Zh. Eksp. Teor. Fiz. 66, 110 (1997) [JETP Lett. 66, 120 (1997)].

19. V. A. Davydov, L. S. Kashevarova, A. V. Rakhmanina, V. Agafonov, R. Ceolin, and H. Szwarc, Carbon 35, 735 (1997).

20. L. S. Fomenko, V. D. Natsik, S. V. Lubenets, V. G. Lirtsman, N. A. Aksenova, A. P. Isakina, A. I. Prokhvatilov, M. A. Strzhemechny, and R. S. Ruoff, Fiz. Nizk. Temp. 21, 465 (1995) [Low Temp. Phys. 21, 364 (1995)].

21. S. V. Lubenets, V. D. Natsik, L. S. Fomenko, A. P. Isakina, A. I. Prokhvatilov, M. A. Strzhemechny, N. A. Aksenova, and R. S. Ruoff, Fiz. Nizk. Temp. 23, 338 (1997) [Low Temp. Phys. 23, 251 (1997)].

22. S. A. Myers, R. A. Assink, J. E. Schirber, and D. A. Loy, Mat. Res. Soc. Symp. Proc., Materials Research Society (1995), 359, p. 505 . 
23. N. P. Kobelev, Ya. M. Soifer, I. O. Bashkin, A. P. Moravsky, and E. G. Ponyatovsky, Mol. Mat. 7, 261 (1996).

24. A. Kailer, Y. G. Gogotsi, and K. G. Nickel, J. Appl. Phys. 81, 3057(1997).

25. E. O. Hall, Proc. Phys. Soc. B64, 747 (1951).

26. J. Friedel, Dislocations, Pergamon Press, Oxford (1964).

27. V. I. Trefilov, Yu. V. Mil'man, S. A. Firstov, Fizicheskije Osnovy Prochnosti Tugoplavkikh Metallov, Kiev, Naukova Dumka (1975).

28. E. O. Hall, Nature 173, 948 (1954).
29. A. S. Taha and F. H. Hammad, Phys. Status. Solidi A119 455 (1990).

30. S. Hoen, N. G. Chopra, X.-D. Xiang, R. Mostovoy, Xianguo Hou, W. A. Vareka, and A. Zettl, Phys. Rev. B46, 12737 (1992).

31. J. E. Schirber, G. K. Kwei, J. D. Jorgensen, R. L. Hitterman, and B. Morosin, Phys. Rev. B51, RC12014 (1995).

32. H. A. Ludwig, W. H. Fietz, F. W. Hornung, K. Grube, B. Wagner, and G. J. Burkhart, Z. Phys. B96, 179 (1994). 